\title{
Risk Factors and Mortality of Carbapenem-Resistant Klebsiella Pneumoniae Bloodstream Infection in a Tertiary-Care Hospital in China: An Eight-Year Retrospective Study
}

Jie Chen

Xiangya Hospital Central South University

Hua Ma

People's Hospital of Liuyang City

Yongfeng Li

Anyang District Hospital

Michal Mastalerz

Comprehensive Pneumology Center

Ting Sheng

People's Hospital of Liuyang City

\section{Xiaoting Xie}

People's Hospital of Ningxiang City

\section{Xiaoming Huang}

Traditional Chinese Medicine Hospital of Leiyang City

\section{Vidya Srokshna}

Comprehensive Pulmonary Center

\section{Ruichao Niu}

Xiangya Hospital Central South University

Pinhua Pan ( $\triangle$ pinhuapan668@csu.edu.cn )

Xiangya Hospital Central South University

\section{Research}

Keywords: Carbapenem resistance, Klebsiella pneumoniae, Bloodstream infection, Risk factors, Mortality, Intensive care units

Posted Date: December 28th, 2020

DOI: https://doi.org/10.21203/rs.3.rs-134806/v1 
License: (c) (i) This work is licensed under a Creative Commons Attribution 4.0 International License. Read Full License 


\section{Abstract}

Background: The prevalence of carbapenem-resistant Klebsiella pneumonia bloodstream infection (CRKP-BSI) is increasing worldwide. CRKP-BSI is associated with high rates of morbidity and mortality due to limited antibiotic choices. Here, we aim to identify the prevalence and risk factors for infection and mortality of CRKP BSI.

Methods: This was a retrospective study of the past data from January $1^{\text {st }}, 2012$ to December $31^{\text {st }}, 2019$ of adult patients with KP-BSI in Xiangya Hospital, China. Data from Demographic and clinical findings were retrieved from medical records.

Results: Among the 706 incidences included in this study, 27.4\% of them (212 / 753) being CR-KP strains. The occurrence of CRKP-BSI was increased from 20.69 to $37.40 \%$ from 2012 to 2019. Hematologic malignancies $(P<0.001$, odds ratio [OR] 4.68, 95\% confidence interval [CI] 2.3-9.4) and ICU acquired infection ( $P=0.003$, OR $2.10,95 \% \mathrm{Cl} 1.3-3.4)$ were identified to be substantial risk factors of carbapenem resistance. The overall 28-day mortality rates of CRKP-BSI patients was significantly higher than that of CSKP-BSI $(P<0.001)$. Logistic regression analysis identified severe sepsis or septic shock incidents (OR, 8.44; $95 \% \mathrm{Cl}, 1.85-38.39)$, inadequate empirical antimicrobial therapy $(\mathrm{OR}, 15.01 ; 95 \% \mathrm{Cl}, 3.70-60.79)$ and corticosteroids use preceding infection onset (OR, 6.45; 95\% $\mathrm{Cl}, 1.12-37.08)$ as the independent predictors of 28-day mortality of CRKP-BSI patients. However, high dose carbapenem combination therapy was identified as anticipated factors of low 28 -day mortality $(\mathrm{OR}, 0.11 ; 95 \% \mathrm{Cl}, 0.03-0.51)$.

Conclusion: The occurrence of CRKP-BSI was significantly increased during the study period. Hematologic malignancies and ICU acquired infection were associated with the development of CRKP BSI. Severe sepsis or septic shock incidents, inadequate empirical antimicrobial therapy and corticosteroids use preceding infection onset caused significant increase of mortality rates in CRKP-BSI patients. High dose carbapenem combination therapy was associated with better outcome.

\section{Introduction}

Klebsiella pneumoniae (KP) is a gram-negative bacteria commonly causing nosocomial infections including pneumonia, bloodstream infections (BSIs), hepatic abscess and urinary tract infections [1-4]. Although $\mathrm{KP}$ is thought to be the second pathogen after Escherichia coli $(E$. coli $)$, that is responsible for gram-negative BSIs in adult patients, recent studies showed the incidence of KP-BSIs had exceeded the incidence of $E$. coli BSIs in intensive care unit (ICU) patients [5, 6]. KP-BSI infections have contributed to high health-care costs and an increased mortality. The crude mortality rate of KP-BSIs patients was reported to span from $20-40 \%$ [6-9].

Carbapenems, a class of broad-spectrum beta-lactam antibiotic drugs for the treatment of many Gramnegative bacteria, have been recommended in first-line therapies for multidrug-resistant KP infections [7]. However, during the last decade, the prevalence of carbapenem-resistant Klebsiella pneumonia (CRKP) is increasing worldwide, such as Israel [4], Europe [5] and some South American countries [6, 7]. [10]. In 
China, according to the data from CHINET (an antimicrobial resistance surveillance network in China) surveillance, the resistance rates of KP to imipenem and meropenem increased from $3.0 \%$ and $2.9 \%$ in 2005 to 26.3 and $25 \%$ in 2018 respectively [11]. Additionally, several reports have shown that resistance to carbapenem is related to the increase of KP-BSIs patients' mortality [12-14]. Therefore, the situation is serious for CRKP-BSI patients.

Due to high morbidity and mortality and lack of appropriate medical intervention, CRKP-BSI has become a great challenge to clinical practitioners $[4,15,16]$. Recognizing the risk factors contributed to the development and mortality of CRKP-BSI may provide the basis for implementing of control measures and therapeutic strategies to prevent the CRKP BSI infection. Thus far, many clinical reports have demonstrated the various risk factors for both development and fatal outcomes of CRKP-BSI infections. For example, the source of skin and soft tissue infection, ICU-acquired infection, central venous catheter, mechanical ventilation, and previous antibiotic exposure were demonstrated to be powerful risk factors leading to the onset of CRKP-BSI $[4,12,17]$, while mechanical ventilation, septic shock, inadequate empirical antibiotic therapy were reported to be independent mortality predictors of CRKP-BSI $[4,12]$. However, the conclusions of these studies were inconsistent.

In this study, we not only evaluate the prevalence of CRKP-BSI in ICU, but also identify the risk factors related to infection and mortality of carbapenem resistance KP-BSI ICU.

\section{Methods}

\section{Study design, setting and patients}

A previous cohort study on patients with KP-BSIs was conducted between 1 January 2012 and 31 December 2019 in Xiangya Hospital, a tertiary healthcare hospital in Changsha, Hunan Province, China. Having approximately 3,500 beds (including 138 beds in 7 ICUs), it is known to be one of the largest comprehensive hospitals in China. This study was approved by the Xiangya Hospital Ethics Committee.

The study comprised of patients aged $\geq 18$ years, who had been admitted to the hospital with KP-BSI during the period. The cases were CRKP-BSI infected patients, and the controls were patients with CSKPBSI. In this report, only the initial positive cultures of KP in bloodstream for each patient were included. Recurrent infections were excluded. Patients with incomplete medical records or polymicrobial BSIs were also excluded. All patients were identified by searching the integrated hospital information system (IHIS), laboratory information system (LIS) and imageology achieving system (RIS) of Xiangya Hospital.

\section{Data Collection}

Results of clinical and microbial characteristics were obtained from the medical records by two experienced respiratory medical doctors. The clinical data collected included: patient demographics (age, gender), comorbidities (congestive heart failure, cerebrovascular disease, chronic lung disease, 
hepatobiliary and pancreatic diseases, kidney diseases, hematologic malignancy, solid tumor, solid organ transplantation, diabetes mellitus, immune diseases), the ward where onset of BSI was identified, previous exposures (previous healthcare interventions, such as hospitalization, surgery, dialysis, endoscopy and mechanical ventilation; previous antibiotics exposures), therapeutic management (choice of antibiotic), and outcomes (span of hospital stay, and mortality at 28 days). In addition, patients general state at the onset of BSI underwent adequate assessment, such as severe sepsis or septic shock. The Charlson comorbidity index $(\mathrm{CCl})$ was used to determine the comorbid conditions as previously described [18]. Acute Physiology and Chronic Health Evaluation II (APACHE II) score was used to calculate the severity of illness within 24 hours following the onset of BSIs. Furthermore, the adequate empirical antimicrobial therapies described by Zarkotou were also taken into consideration [19].

\section{Definitions}

KP-BSI was described to be a positive blood culture of CR-KP strain collected from a patient that showed symptoms and/or signs of the systemic inflammatory response syndrome. For patients that had several incidences of CRKP-BSI, an unusual event was defined as independent occurrence at least 30 days after the final positive blood culture [10]. The date when the blood culture was collected is defined as the onset of BSI. KP-BSIs were classified as healthcare-associated and community-acquired. Ward at the onset of BSI was defined as the first positive blood culture identified more than 2 days after the ward admission without a prior positive blood culture with the same pathogen in last 30 days. BSI sources were established based on the Centers for Disease Control and Prevention criteria [20]. BSI was considered as primary when no source was identified. Septic shock was defined as sepsis associated with organ dysfunction and persistent hypotension despite volume replacement. Combination therapy was defined as a regimen that includes two or more antibiotics, with at least one agent showing in vitro activity against the KP. An empirical antimicrobial therapy was described to be appropriate unless it included at least one drug displaying in vitro activity against the KP isolate, initiated within $48 \mathrm{~h}$ of the index blood culture, and given in adequate doses .

\section{Microbiology}

The Vitek 2 system (BioMérieux, Marcy 1'Étoile, France) was used in the clinical microbiology laboratory for isolate identification and antimicrobial susceptibility testing. KP isolates were considered as carbapenem-resistant KP (CRKP) isolates when they were resistant to one or more carbapenems tested in the clinical microbiology (i.e., ertapenem, imipenem, or meropenem).

\section{Statistical analysis}

SPSS 20.0 (Chicago, IL, USA) was used for statistical analysis. Categorical variables were expressed as frequency counts and percentages with $95 \%$ confidence interval $(95 \% \mathrm{Cl})$. Continuous variables were expressed as median and interquartile ranges (IQRs). Pearson $\chi 2$ or Fisher's exact tests were used to 
analyze categorical variables between groups, and Student's t-test or the Mann-Whitney U test were used to compare continuous variables as appropriate. In analysis of risk factors for CRKP infection and mortality, univariable logistic regression analysis was performed. To identify the independent risk factors, a multivariate logistic regression model was generated to control the effects of confounding variables. Variables with $p$-value $<0.1$ in univariate testing were incorporated into the model using a backward stepwise approach. A two-tailed $p$ value of $<0.05$ was considered statistically significant.

\section{Results}

\section{Incidence and mortality of CRKP-BSIs over the past 8 years}

During the 8-year study period, 706 events of KP-BSI were consecutively collected from 1 January 2012 and 31 December 2019 in Xiangya Hospital, and 30\% (212/706) of these incidences were CRKP isolates. Over the past 8 years, the drug resistance rates of KP detected in blood samples to imipenem and meropenem has been increasing (details are shown in Table 1). 
Table 1

Susceptibility of Klebsiella pneumoniae to antimicrobial agents from 2012 to 2019

\begin{tabular}{|c|c|c|c|c|c|c|c|c|}
\hline $\begin{array}{l}\text { Antimicrobial } \\
\text { agents }\end{array}$ & $\begin{array}{l}2012 \\
(n= \\
58)\end{array}$ & $\begin{array}{l}2013 \\
(n= \\
64)\end{array}$ & $\begin{array}{l}2014 \\
(n= \\
71)\end{array}$ & $\begin{array}{l}2015 \\
(n= \\
83)\end{array}$ & $\begin{array}{l}2016 \\
(n= \\
88)\end{array}$ & $\begin{array}{l}2017 \\
(n= \\
96)\end{array}$ & $\begin{array}{l}2018(n \\
=115)\end{array}$ & $\begin{array}{l}2019(\mathrm{n} \\
=131)\end{array}$ \\
\hline & $\begin{array}{l}\mathrm{R}(\%) \\
\mathrm{S}(\%)\end{array}$ & $\begin{array}{l}\mathrm{R}(\%) \\
\mathrm{S}(\%)\end{array}$ & $\begin{array}{l}\mathrm{R}(\%) \\
\mathrm{S}(\%)\end{array}$ & $\begin{array}{l}\mathrm{R}(\%) \\
\mathrm{S}(\%)\end{array}$ & $\begin{array}{l}\mathrm{R}(\%) \\
\mathrm{S}(\%)\end{array}$ & $\begin{array}{l}R(\%) \\
S(\%)\end{array}$ & $\begin{array}{l}R(\%) \\
S(\%)\end{array}$ & $\begin{array}{l}R(\%) \\
S(\%)\end{array}$ \\
\hline Ampicillin & 78.50 & 79.70 & 81.20 & 85.50 & 86.70 & 87.10 & 88.20 & 88.70 \\
\hline $\begin{array}{l}\text { Ampicillin- } \\
\text { sulbactam }\end{array}$ & $\begin{array}{l}42.7 \\
51.2\end{array}$ & $\begin{array}{l}41.5 \\
53.8\end{array}$ & $\begin{array}{l}45.6 \\
50.3\end{array}$ & $\begin{array}{l}41.3 \\
52.8\end{array}$ & $\begin{array}{l}48.2 \\
49.7\end{array}$ & $\begin{array}{l}47.9 \\
48.8\end{array}$ & $\begin{array}{l}51.2 \\
46.1\end{array}$ & $\begin{array}{l}52.6 \\
44.2\end{array}$ \\
\hline $\begin{array}{l}\text { Piperacillin- } \\
\text { tazobactam }\end{array}$ & $\begin{array}{l}20.1 \\
77.4\end{array}$ & $\begin{array}{l}21.4 \\
76.2\end{array}$ & $\begin{array}{l}21.9 \\
75.2\end{array}$ & $\begin{array}{l}22.3 \\
75.1\end{array}$ & $\begin{array}{l}23.2 \\
73.9\end{array}$ & $\begin{array}{l}23.5 \\
72.8\end{array}$ & $\begin{array}{l}24.1 \\
71.5\end{array}$ & $\begin{array}{l}24.5 \\
71.4\end{array}$ \\
\hline $\begin{array}{l}\text { Cefoperazone- } \\
\text { sulbactam }\end{array}$ & $\begin{array}{l}21.1 \\
76.2\end{array}$ & $\begin{array}{l}27.3 \\
70.8\end{array}$ & $\begin{array}{l}24.2 \\
72.3\end{array}$ & $\begin{array}{l}29.7 \\
64.5\end{array}$ & $\begin{array}{l}25.3 \\
71.1\end{array}$ & $\begin{array}{l}23.5 \\
71.4\end{array}$ & $\begin{array}{l}22.7 \\
72.5\end{array}$ & $\begin{array}{l}22.1 \\
72.8\end{array}$ \\
\hline Cefazolin & $\begin{array}{l}44.3 \\
52.8\end{array}$ & $\begin{array}{l}43.7 \\
53.5\end{array}$ & $\begin{array}{l}51.0 \\
49.0\end{array}$ & $\begin{array}{l}42.7 \\
57.3\end{array}$ & $\begin{array}{l}51.2 \\
48.8\end{array}$ & $\begin{array}{l}46.5 \\
53.5\end{array}$ & $\begin{array}{l}49.7 \\
50.3\end{array}$ & $\begin{array}{l}48.2 \\
51.8\end{array}$ \\
\hline Cefuroxime & $\begin{array}{l}45.8 \\
52.4\end{array}$ & $\begin{array}{l}43.6 \\
54.8\end{array}$ & $\begin{array}{l}48.5 \\
50.3\end{array}$ & $\begin{array}{l}47.2 \\
51.4\end{array}$ & $\begin{array}{l}46.7 \\
52.5\end{array}$ & $\begin{array}{l}47.4 \\
51.8\end{array}$ & $\begin{array}{l}48.2 \\
50.5\end{array}$ & $\begin{array}{l}49.1 \\
48.9\end{array}$ \\
\hline Ceftazidime & $\begin{array}{l}24.7 \\
72.8\end{array}$ & $\begin{array}{l}25.5 \\
71.3\end{array}$ & $\begin{array}{l}25.3 \\
71.4\end{array}$ & $\begin{array}{l}28.5 \\
70.1\end{array}$ & $\begin{array}{l}29.0 \\
68.9\end{array}$ & $\begin{array}{l}30.4 \\
67.8\end{array}$ & $\begin{array}{l}31.5 \\
64.8\end{array}$ & $\begin{array}{l}32.4 \\
63.3\end{array}$ \\
\hline Ceftriaxone & $\begin{array}{l}38.9 \\
61.1\end{array}$ & $\begin{array}{l}39.7 \\
60.3\end{array}$ & $\begin{array}{l}41.2 \\
58.8\end{array}$ & $\begin{array}{l}42.4 \\
57.6\end{array}$ & $\begin{array}{l}43.3 \\
56.7\end{array}$ & $\begin{array}{l}44.7 \\
55.3\end{array}$ & $\begin{array}{l}46.3 \\
53.7\end{array}$ & $\begin{array}{l}47.1 \\
52.9\end{array}$ \\
\hline Cefepime & $\begin{array}{l}28.3 \\
70.4\end{array}$ & $\begin{array}{l}29.4 \\
69.5\end{array}$ & $\begin{array}{l}30.7 \\
67.8\end{array}$ & $\begin{array}{l}28.6 \\
70.6\end{array}$ & $\begin{array}{l}29.5 \\
69.1\end{array}$ & $\begin{array}{l}28.6 \\
70.1\end{array}$ & $\begin{array}{l}28.9 \\
70.0\end{array}$ & $\begin{array}{l}27.7 \\
71.6\end{array}$ \\
\hline Cefotetan & $\begin{array}{l}17.5 \\
80.7\end{array}$ & $\begin{array}{l}18.8 \\
80.1\end{array}$ & $\begin{array}{l}17.9 \\
80.7\end{array}$ & $\begin{array}{l}18.4 \\
80.4\end{array}$ & $\begin{array}{l}17.3 \\
81.1\end{array}$ & $\begin{array}{l}18.1 \\
80.2\end{array}$ & $\begin{array}{l}18.5 \\
80.2\end{array}$ & $\begin{array}{l}19.7 \\
78.6\end{array}$ \\
\hline Aztreonam & $\begin{array}{l}37.9 \\
62.1\end{array}$ & $\begin{array}{l}37.6 \\
62.4\end{array}$ & $\begin{array}{l}37.7 \\
62.3\end{array}$ & $\begin{array}{l}37.5 \\
62.5\end{array}$ & $\begin{array}{l}38.0 \\
62.0\end{array}$ & $\begin{array}{l}36.8 \\
63.2\end{array}$ & $\begin{array}{l}37.3 \\
62.7\end{array}$ & $\begin{array}{l}38.2 \\
61.8\end{array}$ \\
\hline Ertapenem & $\begin{array}{l}18.1 \\
81.9\end{array}$ & $\begin{array}{l}18.5 \\
81.5\end{array}$ & $\begin{array}{l}18.8 \\
81.2\end{array}$ & $\begin{array}{l}18.9 \\
81.1\end{array}$ & $\begin{array}{l}19.3 \\
80.7\end{array}$ & $\begin{array}{l}19.8 \\
80.2\end{array}$ & $\begin{array}{l}20.0 \\
80.0\end{array}$ & $\begin{array}{l}21.9 \\
79.1\end{array}$ \\
\hline Imipenem & $\begin{array}{l}17.0 \\
83.0\end{array}$ & $\begin{array}{l}17.1 \\
82.9\end{array}$ & $\begin{array}{l}17.3 \\
82.7\end{array}$ & $\begin{array}{l}17.5 \\
82.5\end{array}$ & $\begin{array}{l}18.1 \\
81.9\end{array}$ & $\begin{array}{l}18.3 \\
81.7\end{array}$ & $\begin{array}{l}18.8 \\
81.2\end{array}$ & $\begin{array}{l}19.5 \\
80.5\end{array}$ \\
\hline Meropenem & $\begin{array}{l}16.9 \\
83.1\end{array}$ & $\begin{array}{l}17.0 \\
83.0\end{array}$ & $\begin{array}{l}17.1 \\
82.9\end{array}$ & $\begin{array}{l}17.2 \\
82.8\end{array}$ & $\begin{array}{l}17.9 \\
82.1\end{array}$ & $\begin{array}{l}18.7 \\
81.3\end{array}$ & $\begin{array}{l}18.5 \\
82.5\end{array}$ & $\begin{array}{l}19.2 \\
81.8\end{array}$ \\
\hline Amikacin & $\begin{array}{l}13.8 \\
86.2\end{array}$ & $\begin{array}{l}13.5 \\
86.5\end{array}$ & $\begin{array}{l}13.3 \\
86.7\end{array}$ & $\begin{array}{l}13.1 \\
86.9\end{array}$ & $\begin{array}{l}12.5 \\
87.5\end{array}$ & $\begin{array}{l}12.4 \\
87.6\end{array}$ & $\begin{array}{l}11.8 \\
88.2\end{array}$ & $\begin{array}{l}11.2 \\
88.8\end{array}$ \\
\hline Gentamicin & $\begin{array}{l}29.4 \\
69.4\end{array}$ & $\begin{array}{l}28.3 \\
70.1\end{array}$ & $\begin{array}{l}28.0 \\
70.7\end{array}$ & $\begin{array}{l}27.4 \\
71.6\end{array}$ & $\begin{array}{l}23.8 \\
75.0\end{array}$ & $\begin{array}{l}23.2 \\
75.4\end{array}$ & $\begin{array}{l}23.4 \\
75.1\end{array}$ & $\begin{array}{l}22.8 \\
75.7\end{array}$ \\
\hline
\end{tabular}

R Resistance, S susceptible, SMZ-TMP Trimethoprim-sulfamethoxazole 


\begin{tabular}{|c|c|c|c|c|c|c|c|c|}
\hline \multirow[t]{2}{*}{$\begin{array}{l}\text { Antimicrobial } \\
\text { agents }\end{array}$} & $\begin{array}{l}2012 \\
(n= \\
58)\end{array}$ & $\begin{array}{l}2013 \\
(n= \\
64)\end{array}$ & $\begin{array}{l}2014 \\
(n= \\
71)\end{array}$ & $\begin{array}{l}2015 \\
(n= \\
83)\end{array}$ & $\begin{array}{l}2016 \\
(n= \\
88)\end{array}$ & $\begin{array}{l}2017 \\
(n= \\
96)\end{array}$ & $\begin{array}{l}2018(n \\
=115)\end{array}$ & $\begin{array}{l}2019(n \\
=131)\end{array}$ \\
\hline & $\begin{array}{l}\mathrm{R}(\%) \\
\mathrm{S}(\%)\end{array}$ & $\begin{array}{l}\mathrm{R}(\%) \\
\mathrm{S}(\%)\end{array}$ & $\begin{array}{l}\mathrm{R}(\%) \\
\mathrm{S}(\%)\end{array}$ & $\begin{array}{l}\mathrm{R}(\%) \\
\mathrm{S}(\%)\end{array}$ & $\begin{array}{l}\mathrm{R}(\%) \\
\mathrm{S}(\%)\end{array}$ & $\begin{array}{l}\mathrm{R}(\%) \\
\mathrm{S}(\%)\end{array}$ & $\begin{array}{l}\mathrm{R}(\%) \\
\mathrm{S}(\%)\end{array}$ & $\begin{array}{l}\mathrm{R}(\%) \\
\mathrm{S}(\%)\end{array}$ \\
\hline Tobramycin & $\begin{array}{l}18.1 \\
71.5\end{array}$ & $\begin{array}{l}16.4 \\
74.2\end{array}$ & $\begin{array}{l}15.5 \\
74.9\end{array}$ & $\begin{array}{l}17.2 \\
72.4\end{array}$ & $\begin{array}{l}14.7 \\
76.1\end{array}$ & $\begin{array}{l}16.5 \\
73.8\end{array}$ & $\begin{array}{l}15.3 \\
74.5\end{array}$ & $\begin{array}{l}14.9 \\
73.2\end{array}$ \\
\hline Ciprofloxacin & $\begin{array}{l}38.2 \\
57.5\end{array}$ & $\begin{array}{l}37.6 \\
58.9\end{array}$ & $\begin{array}{l}39.7 \\
56.4\end{array}$ & $\begin{array}{l}37.8 \\
58.1\end{array}$ & $\begin{array}{l}34.8 \\
61.2\end{array}$ & $\begin{array}{l}35.7 \\
60.1\end{array}$ & $\begin{array}{l}32.1 \\
62.3\end{array}$ & $\begin{array}{l}33.7 \\
61.5\end{array}$ \\
\hline Levofloxacin & $\begin{array}{l}28.5 \\
67.3\end{array}$ & $\begin{array}{l}25.4 \\
70.7\end{array}$ & $\begin{array}{l}25.8 \\
70.3\end{array}$ & $\begin{array}{l}24.6 \\
71.9\end{array}$ & $\begin{array}{l}31.5 \\
65.7\end{array}$ & $\begin{array}{l}29.4 \\
67.2\end{array}$ & $\begin{array}{l}28.7 \\
68.8\end{array}$ & $\begin{array}{l}28.9 \\
68.5\end{array}$ \\
\hline SMZ-TMP & $\begin{array}{l}31.3 \\
78.7\end{array}$ & $\begin{array}{l}31.8 \\
78.2\end{array}$ & $\begin{array}{l}32.4 \\
67.6\end{array}$ & $\begin{array}{l}35.2 \\
64.8\end{array}$ & $\begin{array}{l}35.8 \\
64.2\end{array}$ & $\begin{array}{l}36.6 \\
63.4\end{array}$ & $\begin{array}{l}38.2 \\
61.8\end{array}$ & $\begin{array}{l}37.5 \\
62.5\end{array}$ \\
\hline Tigecycline & & & & & 097.7 & $\begin{array}{l}1.2 \\
95.8\end{array}$ & 2.494 .3 & 3.992 .6 \\
\hline
\end{tabular}

The percentage of KP in blood sample from 2012 to 2019 fluctuated from 5.3 to $7 \%$ during the 8 years (5.3 in 2012, 5.7 in 2013, 5.2 in 2014, 5.9 in 2015, 6.1 in 2016, 6.3 in 2017, 6.5 in 2018 and 7 in 2019). The percentage of CRKP in KP BSI from 2012 to 2019 were 20.69, 21.88, 22.54, 27.71, 30.68, 32.29, 34.78 and $37.40 \%$ respectively, which rose fastest from 2015 to 2019, as shown in Fig. 1. Additionally, the total 28day mortality rate of KP-BSI patients was $26.1 \%$, and it was higher in patients with CRKP-BSIs than in those with CSKP-BSIs ( $42.5 \%$ vs. $20.2 \%$, respectively, $P<0.001$ ) (Fig. 1 ). The mortality of KP BSI has increased from $17.24 \%$ in 2012 to $27.45 \%$ in 2019 . The mortality of CRKP BSI and CSKP BSI increased from 33.33 and $13.04 \%$ in 2012 to 42.86 and $19.51 \%$ in 2019 , respectively (Fig. 1).

\section{Clinical Characteristics Of Patients}

The average age of these 706 patients was 58.9 years (range: 18-92 years), and the male patients accounted for $66.9 \%(472 / 706)$. The most frequent comorbidities of these patients were kidney diseases (172/706, 24.4\%), chronic lung diseases (146/706, 20.7\%) and diabetes mellitus (140/706, 19.8\%). The number of KP-BSI patients with the Carlson comorbidity index (CCI) $\geq 3$ was 348 (49.3\%). Most of the KPBSIs cases were acquired at ICU $(300 / 706,42.5 \%)$ and most of them were undergoing hospitalization (378/706, 53.5\%) within 12 months preceding infection onset. The most common KP infection source was primary bloodstream infections $(408 / 706,57.8 \%)$. Additionally, KP could be detected in samples from other sites (348/706, 49.3\%), including pulmonary $(166 / 706,23.5 \%)$, pancreaticobiliary tract infection $(40 / 706,5.7 \%)$ and urinary tract infection (35/706, 5.0\%), etc. 138 out of 706 (19.5\%) KP-BSI patients (19.5\%) were treated with corticosteroids and 150 out of 706 (21.2\%) KP-BSI patients underwent 
chemotherapy or radiotherapy. 261 out of 706 (37.0\%) KP-BSI patients did not receive adequate empirical antibiotic therapy. The number of KP-BSI patients with APACHE II score more than 15 was 240 (34.0\%).

\section{Risk Factors For The Development Of Crkp}

We identified the clinical characteristics affecting the development of CRKP-BSIs, by comparing patient demographics, clinical characteristics, type of infections, and prior antibiotic exposures of patients with CRKP-BSIs and CSKP-BSIs (Table 2). The factors determined to be more relevantly related with CRKPBSIs, using the univariable logistic regression analysis, included hematologic malignancy, ICU-acquired infection, undergoing hospitalization within 12 months preceding infection onset, undergoing surgery within 30 days preceding infection onset, inadequate empirical antibiotic therapy, corticosteroids use preceding infection onset and probable pulmonary source of infection. Using multivariate logistic regression analysis, hematologic malignancy (Odds ratio (OR) 4.68, 95\% Cl 2.3-9.4) and ICU-acquired infection (OR 2.10, 95\% Cl 1.3-3.4) was identified to be independent factors for CRKP-BSI development (Table 3). 
Table 2

Comparison of clinical characteristics between patients with CRKP-BSI and CSKP-BSI.

\begin{tabular}{|c|c|c|c|c|}
\hline Variable & $\begin{array}{l}\text { The total }(\mathrm{N}= \\
706)\end{array}$ & $\begin{array}{l}\text { CRKP }(N= \\
212)\end{array}$ & $\begin{array}{l}\operatorname{CSKP}(N= \\
494)\end{array}$ & $\begin{array}{l}P \\
\text { value }\end{array}$ \\
\hline Male, $n,(\%)$ & $472(66.9)$ & $138(65.1)$ & $334(67.6)$ & 0.515 \\
\hline $\operatorname{Age}(y)$, mean $\pm S D$ & $58.9 \pm 16.0$ & $60.8 \pm 16.0$ & $58.1 \pm 16.2$ & 0.147 \\
\hline \multicolumn{5}{|l|}{ Comorbidities- No., \% } \\
\hline Congestive heart failure & $96(13.6)$ & $22(10.4)$ & $74(15.0)$ & 0.102 \\
\hline Cerebrovascular disease & $122(17.3)$ & $40(18.9)$ & $82(16.6)$ & 0.465 \\
\hline Chronic lung diseases & $146(20.7)$ & $52(24.5)$ & $94(19.0)$ & 0.098 \\
\hline Hepatobiliary and pancreatic diseases & $130(18.4)$ & $30(14.2)$ & $100(20.2)$ & 0.056 \\
\hline Kidney diseases & $172(24.4)$ & $56(26.4)$ & $116(23.5)$ & 0.405 \\
\hline Hematologic malignancy & $84(11.9)$ & $54(25.5)$ & $30(6.1)$ & $\dot{0} 001$ \\
\hline Solid tumor & $118(16.7)$ & $40(18.9)$ & $78(15.8)$ & 0.315 \\
\hline Solid organ transplantation & $49(6.9)$ & $19(9.0)$ & $30(6.1)$ & 0.166 \\
\hline Diabetes mellitus & $140(19.8)$ & $36(17.0)$ & $104(21.1)$ & 0.214 \\
\hline Immune diseases & $42(5.9)$ & $18(8.5)$ & $24(4.9)$ & 0.061 \\
\hline Charlson comorbidity index $\geq 3$ & $348(49.3)$ & $112(52.8)$ & $236(47.8)$ & 0.218 \\
\hline \multicolumn{5}{|l|}{$\begin{array}{l}\text { Pre-infection healthcare interventions- } \\
\text { No., \% }\end{array}$} \\
\hline Enema $^{\mathrm{c}}$ & $52(7.4)$ & $18(8.5)$ & $34(6.9)$ & 0.453 \\
\hline Nasogastric catheter ${ }^{b}$ & $174(24.6)$ & $46(21.7)$ & $128(25.9)$ & 0.234 \\
\hline Urinary catheter ${ }^{b}$ & $254(36.0)$ & $82(38.7)$ & $172(34.8)$ & 0.327 \\
\hline Surgical drain ${ }^{b}$ & $104(14.7)$ & $38(17.9)$ & $66(13.4)$ & 0.117 \\
\hline Central venous catheter ${ }^{b}$ & $190(26.9)$ & $64(30.2)$ & $126(25.5)$ & 0.198 \\
\hline Peripheral arterial catheter ${ }^{b}$ & $65(9.2)$ & $26(12.3)$ & $39(7.9)$ & 0.066 \\
\hline \multicolumn{5}{|c|}{ a During the 12 months preceding infection onset. } \\
\hline \multicolumn{5}{|c|}{${ }^{b}$ During the 30 days preceding infection onset. } \\
\hline
\end{tabular}




\begin{tabular}{|c|c|c|c|c|}
\hline Variable & $\begin{array}{l}\text { The total }(N= \\
706)\end{array}$ & $\begin{array}{l}\text { CRKP }(N= \\
212)\end{array}$ & $\begin{array}{l}\operatorname{CSKP}(N= \\
494)\end{array}$ & $\begin{array}{l}P \\
\text { value }\end{array}$ \\
\hline Blood purification ${ }^{b}$ & $72(10.2)$ & $28(13.2)$ & $44(8.9)$ & 0.083 \\
\hline Tracheal cannula ${ }^{b}$ & $288(40.8)$ & $91(42.9)$ & $197(39.9)$ & 0.450 \\
\hline Tracheostomy ${ }^{b}$ & $54(7.6)$ & $20(9.4)$ & $34(6.9)$ & 0.242 \\
\hline Mechanical ventilation ${ }^{c}$ & $182(25.8)$ & $64(30.2)$ & $118(23.9)$ & 0.079 \\
\hline Gastroscopy ${ }^{c}$ & $15(2.1)$ & $6(2.8)$ & $9(1.8)$ & 0.394 \\
\hline Colonoscopy ${ }^{\mathrm{c}}$ & $6(0.8)$ & $2(0.9)$ & $4(0.8)$ & 0.859 \\
\hline Bronchoscopy ${ }^{c}$ & $78(11.0)$ & $24(11.3)$ & $54(10.9)$ & 0.880 \\
\hline Sputum suction ${ }^{b}$ & $356(50.4)$ & $115(54.2)$ & $241(48.8)$ & 0.184 \\
\hline Thoracentesis ${ }^{b}$ & $56(7.9)$ & $21(9.9)$ & $35(7.1)$ & 0.204 \\
\hline Abdominocentesis ${ }^{b}$ & $35(5.0)$ & $12(5.7)$ & $23(4.7)$ & 0.573 \\
\hline Bone marrow puncture ${ }^{b}$ & $91(12.9)$ & $33(15.6)$ & $58(11.7)$ & 0.164 \\
\hline Lumbar puncture $^{\mathrm{b}}$ & $68(9.6)$ & $25(11.8)$ & $43(8.7)$ & 0.202 \\
\hline Previous surgery ${ }^{b}$ & $140(19.8)$ & $58(27.4)$ & $82(16.6)$ & 0.001 \\
\hline Parenteral nutrition ${ }^{\mathrm{b}}$ & $237(33.6)$ & $77(36.3)$ & $160(32.4)$ & 0.311 \\
\hline Previous hospitalization ${ }^{a}$ & $378(53.5)$ & $134(63.2)$ & $244(49.4)$ & 0.001 \\
\hline \multicolumn{5}{|l|}{$\begin{array}{l}\text { Previous treatments administered }{ }^{\text {b_ }} \\
\text { No., \% }\end{array}$} \\
\hline Corticosteroids & $138(19.5)$ & $52(24.5)$ & $86(17.4)$ & 0.029 \\
\hline Chemotherapy or radiotherapy & $150(21.2)$ & $40(18.9)$ & $110(22.3)$ & 0.311 \\
\hline Inadequate empirical antibiotic therapy & $261(37.0)$ & $96(45.3)$ & $165(33.4)$ & 0.003 \\
\hline \multicolumn{5}{|l|}{ Previous use of antibiotics } \\
\hline Carbapenems & $218(30.9)$ & $68(32.1)$ & $150(30.4)$ & 0.652 \\
\hline \multicolumn{5}{|c|}{ a During the 12 months preceding infection onset. } \\
\hline \multicolumn{5}{|c|}{${ }^{b}$ During the 30 days preceding infection onset. } \\
\hline
\end{tabular}




\begin{tabular}{|c|c|c|c|c|}
\hline Variable & $\begin{array}{l}\text { The total }(\mathrm{N}= \\
706)\end{array}$ & $\begin{array}{l}\text { CRKP }(N= \\
212)\end{array}$ & $\begin{array}{l}\operatorname{CSKP}(N= \\
494)\end{array}$ & $\begin{array}{l}P \\
\text { value }\end{array}$ \\
\hline Glycopeptides & $144(20.4)$ & $48(22.6)$ & $96(19.4)$ & 0.332 \\
\hline Quinolones & $227(32.2)$ & $74(34.9)$ & $153(31.0)$ & 0.305 \\
\hline 3rd/4th generation cephalosporins & $102(14.4)$ & $38(17.9)$ & $64(13.0)$ & 0.085 \\
\hline 1st/2nd generation cephalosporins & $86(12.2)$ & $25(11.8)$ & $61(12.3)$ & 0.836 \\
\hline Penicillins & $35(5.0)$ & $8(3.8)$ & $27(5.5)$ & 0.342 \\
\hline$\beta$-lactamase inhibitor & $308(43.6)$ & $101(47.6)$ & 207 (41.9) & 0.159 \\
\hline Aminoglycosides & $27(3.8)$ & $12(5.7)$ & $15(3.0)$ & 0.096 \\
\hline Linezolid & $41(5.8)$ & $14(6.6)$ & $27(5.5)$ & 0.553 \\
\hline Tigecycline & 77 (10.9) & $28(13.2)$ & $49(9.9)$ & 0.199 \\
\hline Daptomycin & $8(1.1)$ & $2(0.9)$ & $6(1.2)$ & 0.755 \\
\hline Nitroimidazoles & $32(4.5)$ & $13(6.1)$ & $19(3.8)$ & 0.181 \\
\hline \multicolumn{5}{|l|}{ Source of BSI- No., \% } \\
\hline Primary & $408(57.8)$ & $112(52.8)$ & $296(59.9)$ & 0.080 \\
\hline $\begin{array}{l}\text { KP detection in samples from other } \\
\text { sites }\end{array}$ & $348(49.3)$ & $116(54.7)$ & $232(47.0)$ & 0.059 \\
\hline Pulmonary & $166(23.5)$ & $66(31.7)$ & $100(20.1)$ & 0.001 \\
\hline Pleural effusion & $16(2.3)$ & $6(2.8)$ & $10(2.0)$ & 0.510 \\
\hline Pancreaticobiliary tract infection & $40(5.7)$ & $14(6.6)$ & $26(5.3)$ & 0.480 \\
\hline Live abscess & $30(4.2)$ & $12(5.7)$ & $18(3.6)$ & 0.223 \\
\hline Urinary tract infection & $35(5.0)$ & $11(5.2)$ & $24(4.9)$ & 0.853 \\
\hline Intestinal infection & $26(3.7)$ & $6(2.8)$ & $20(4.0)$ & 0.431 \\
\hline Intra-abdominal infection & $17(2.4)$ & $5(2.4)$ & $12(2.4)$ & 0.955 \\
\hline Skin infection & $12(1.7)$ & $4(1.9)$ & $8(1.6)$ & 0.801 \\
\hline Cerebrospinal fluid & $6(0.8)$ & $2(0.9)$ & $4(0.8)$ & 0.859 \\
\hline
\end{tabular}

a During the 12 months preceding infection onset.

${ }^{b}$ During the 30 days preceding infection onset.

${ }^{\mathrm{c}}$ During the 72 hours preceding infection onset. 


\begin{tabular}{|lcccc|}
\hline Variable & $\begin{array}{c}\text { The total }(\mathrm{N}= \\
\text { 706) }\end{array}$ & $\begin{array}{l}\text { CRKP }(\mathrm{N}= \\
\text { 212) }\end{array}$ & $\begin{array}{l}\text { CSKP }(\mathrm{N}= \\
\text { 494) }\end{array}$ & $\begin{array}{l}\boldsymbol{P} \\
\text { value }\end{array}$ \\
\hline Ward at the onset of BSI- No., \% & & & \\
\hline Intensive care units & $300(42.5)$ & $120(56.6)$ & $180(36.4)$ & 0.001 \\
\hline Medical wards & $232(32.9)$ & $54(25.5)$ & $178(36.0)$ & 0.006 \\
\hline Surgical wards & $170(24.1)$ & $38(17.9)$ & $132(26.7)$ & 0.012 \\
\hline Severity at BSI onset- No., \% & & & \\
\hline APACHE II score > 15 & $240(34.0)$ & $86(40.6)$ & $154(31.2)$ & 0.016 \\
\hline Severe sepsis / septic shock & $244(34.6)$ & $88(41.5)$ & $156(31.6)$ & 0.011 \\
\hline Outcome- No., \% & & & \\
\hline 28-day mortality & & & \\
\hline
\end{tabular}

Table 3

Multivariate analysis of factors leading to the development of CRKP-BSI

\begin{tabular}{|llll|}
\hline Variable & Pvalue & OR & $95 \% \mathrm{Cl}$ \\
\hline Hematologic malignancy & $<0.001$ & 4.68 & $2.322-9.427$ \\
\hline Intensive Care Units acquired infection & 0.003 & 2.101 & $1.291-3.420$ \\
\hline Previous hospitalization ${ }^{\text {a }}$ & 0.761 & 1.097 & $0.603-1.996$ \\
\hline Previous surgery ${ }^{\text {b }}$ & 0.247 & 1.464 & $0.767-2.794$ \\
\hline Inadequate empirical antibiotic therapy ${ }^{\text {b }}$ & 0.150 & 1.485 & $0.866-2.546$ \\
\hline Corticosteroids use preceding infection onset ${ }^{\text {b }}$ & 0.083 & 1.708 & $0.933-3.125$ \\
\hline Pulmonary source of BSI & 0.061 & 1.748 & $0.975-3.133$ \\
\hline a During the 12 months preceding infection onset. & & \\
\hline b During the 30 days preceding infection onset. & & & \\
\hline c During the 72 hours preceding infection onset. & & & \\
\hline
\end{tabular}




\section{Risk Factors For Mortality Of Crkp-bsi Patients}

Overall, the 28-day mortality of CRKP-BSI patients was $42.5 \%$. In order to identify the risk factors correlated with crude 28-day mortality of CRKP-BSI patients, we compared patient demographics, clinical characteristics, type of infections, and prior antibiotic exposures of deceased and survivor patients of CRKP-BSIs (Table 4). In the univariable logistic regression analysis, congestive heart failure and ICUacquired infection were determined to be the main risk factors. Besides, the mortality rate was also affected by various events preceding CRKP-BSI onset, such as blood purification, mechanical ventilation, corticosteroids use preceding infection onset. Additionally, inadequate empirical antibiotic therapy, high APACHE II score, severe sepsis / septic shock were associated with the crude 28-day mortality in univariable logistic regression analysis, In contrast, mortality was lower in patients that underwent high doses of carbapenem combination therapy. In the final multivariable logistic regression analysis model, only corticosteroids use preceding infection onset (OR 6.45, 95\% $\mathrm{Cl} 1.12-37.08, P=0.037$ ), inadequate empirical antibiotic therapy (OR 15.01,95\% Cl 3.70-60.79, $P<0.001$ ), severe sepsis / septic shock (OR $8.44,95 \% \mathrm{Cl} 1.84-38.39, P=0.006)$ remained adversely and independently associated with 28-days mortality, whereas a protective effect was observed for high doses of carbapenem combination therapy (OR $0.11,95 \% \mathrm{Cl} 0.03-0.51, P=0.004$ ) (Table 5). 
Table 4

Univariate analysis of factors for 28-day mortality in patients with infections caused by CRKP-BSI

\begin{tabular}{|c|c|c|c|}
\hline Variable & Death $(\mathrm{N}=90)$ & Survivors $(\mathrm{N}=122)$ & $P$ value \\
\hline Male, n, (\%) & $56(62.2)$ & $82(67.2)$ & 0.451 \\
\hline $\operatorname{Age}(y)$, mean $\pm S D$ & $61.1 \pm 17.7$ & $60.7 \pm 14.8$ & 0.900 \\
\hline \multicolumn{4}{|l|}{ Comorbidities- No., \% } \\
\hline Congestive heart failure & $14(15.6)$ & $8(6.6)$ & 0.034 \\
\hline Cerebrovascular disease & $18(20.0)$ & $22(18.0)$ & 0.717 \\
\hline Chronic lung diseases & $22(24.4)$ & $30(24.6)$ & 0.981 \\
\hline Hepatobiliary and pancreatic diseases & $17(18.9)$ & $13(10.7)$ & 0.089 \\
\hline Kidney diseases & $24(26.7)$ & $32(26.2)$ & 0.943 \\
\hline Hematologic malignancy & $26(28.9)$ & $28(23.0)$ & 0.327 \\
\hline Solid tumor & $14(15.6)$ & $26(21.3)$ & 0.290 \\
\hline Solid organ transplantation & $9(10.0)$ & $10(8.2)$ & 0.650 \\
\hline Diabetes mellitus & $12(13.3)$ & $24(19.7)$ & 0.224 \\
\hline Immune diseases & $10(11.1)$ & $8(6.6)$ & 0.240 \\
\hline Charlson comorbidity index $\geq 3$ & $46(51.1)$ & $66(54.1)$ & 0.667 \\
\hline \multicolumn{4}{|c|}{ Pre-infection healthcare interventions- No., \% } \\
\hline Enema $^{\mathrm{c}}$ & $9(10.0)$ & $9(7.4)$ & 0.498 \\
\hline Nasogastric catheter ${ }^{b}$ & $14(15.6)$ & $32(26.2)$ & 0.062 \\
\hline Urinary catheter ${ }^{b}$ & $34(37.8)$ & $48(39.3)$ & 0.817 \\
\hline Surgical drain ${ }^{b}$ & $16(17.8)$ & $22(18.0)$ & 0.962 \\
\hline Central venous catheter ${ }^{b}$ & $30(33.3)$ & $34(27.9)$ & 0.392 \\
\hline Peripheral arterial catheter ${ }^{b}$ & $15(16.7)$ & $11(9.0)$ & 0.093 \\
\hline Blood purification ${ }^{b}$ & $19(21.1)$ & $9(7.4)$ & 0.004 \\
\hline
\end{tabular}

${ }^{a}$ During the 12 months preceding infection onset.

${ }^{b}$ During the 30 days preceding infection onset.

${ }^{\mathrm{C}}$ During the 72 hours preceding infection onset. 


\begin{tabular}{|c|c|c|c|}
\hline Variable & Death $(\mathrm{N}=90)$ & Survivors ( $N=122)$ & $P$ value \\
\hline Tracheal cannula ${ }^{b}$ & $47(48.9)$ & $44(38.5)$ & 0.132 \\
\hline Tracheostomy ${ }^{b}$ & $11(12.2)$ & $9(7.4)$ & 0.233 \\
\hline Mechanical ventilation ${ }^{c}$ & $36(40.0)$ & $28(23.0)$ & 0.008 \\
\hline Gastroscopy c & $3(3.3)$ & $3(2.5)$ & 0.704 \\
\hline Colonoscopy ${ }^{\mathrm{c}}$ & $2(2.2)$ & $1(0.8)$ & 0.393 \\
\hline Bronchoscopy ${ }^{c}$ & $14(15.6)$ & $10(8.2)$ & 0.095 \\
\hline Sputum suction ${ }^{b}$ & $52(57.8)$ & $63(51.6)$ & 0.375 \\
\hline Thoracentesis ${ }^{b}$ & $11(12.2)$ & $10(8.2)$ & 0.332 \\
\hline Abdominocentesis ${ }^{b}$ & $6(6.7)$ & $6(5.7)$ & 0.586 \\
\hline Bone marrow puncture ${ }^{b}$ & $14(15.6)$ & 19 (15.6) & 0.997 \\
\hline Lumbar puncture ${ }^{b}$ & $11(12.2)$ & $14(11.5)$ & 0.868 \\
\hline Previous surgery ${ }^{b}$ & $26(28.9)$ & $32(26.2)$ & 0.668 \\
\hline Parenteral nutrition ${ }^{b}$ & $37(41.1)$ & $40(32.8)$ & 0.213 \\
\hline Previous hospitalization ${ }^{a}$ & $60(66.7)$ & $74(60.7)$ & 0.370 \\
\hline \multicolumn{4}{|l|}{ Previous treatments administered ${ }^{\mathrm{b}}$ - No., $\%$} \\
\hline Corticosteroids & $38(42.2)$ & $14(11.5)$ & $<0.001$ \\
\hline Chemotherapy or radiotherapy & $12(13.3)$ & $28(23.0)$ & 0.077 \\
\hline Inadequate empirical antibiotic therapy & $78(86.7)$ & $18(14.8)$ & $<0.001$ \\
\hline \multicolumn{4}{|l|}{ Previous use of antibiotics } \\
\hline Carbapenems & $30(33.3)$ & $38(31.1)$ & 0.736 \\
\hline Glycopeptides & $22(24.4)$ & $26(21.3)$ & 0.590 \\
\hline Quinolones & $34(37.8)$ & $40(32.8)$ & 0.451 \\
\hline \multicolumn{4}{|c|}{ a During the 12 months preceding infection onset. } \\
\hline \multicolumn{4}{|c|}{${ }^{b}$ During the 30 days preceding infection onset. } \\
\hline${ }^{\mathrm{C}}$ During the 72 hours preceding infectic & & & \\
\hline
\end{tabular}




\begin{tabular}{|c|c|c|c|}
\hline Variable & Death $(N=90)$ & Survivors $(N=122)$ & $P$ value \\
\hline 3rd/4th generation cephalosporins & $14(15.6)$ & $24(19.7)$ & 0.440 \\
\hline 1st/2nd generation cephalosporins & $10(11.1)$ & $15(12.3)$ & 0.792 \\
\hline Penicillin & $4(4.4)$ & $4(3.3)$ & 0.660 \\
\hline$\beta$-lactamase inhibitor & $49(54.4)$ & $52(42.6)$ & 0.088 \\
\hline Aminoglycosides & $5(5.6)$ & $7(5.7)$ & 0.995 \\
\hline Linezolid & $4(4.4)$ & $10(8.2)$ & 0.277 \\
\hline Tigecycline & $13(14.4)$ & $15(12.3)$ & 0.648 \\
\hline Daptomycin & $1(1.1)$ & $1(0.8)$ & 0.828 \\
\hline Nitroimidazoles & $6(6.7)$ & $7(5.7)$ & 0.781 \\
\hline \multicolumn{4}{|l|}{ Source of BSI- No., \% } \\
\hline Primary & $44(48.9)$ & $68(55.7)$ & 0.323 \\
\hline KP detection in samples from other sites & $55(61.1)$ & $61(50.0)$ & 0.108 \\
\hline Pulmonary & $20(22.2)$ & $36(29.5)$ & 0.234 \\
\hline Pleural effusion & $2(2.2)$ & $4(3.3)$ & 0.647 \\
\hline Pancreaticobiliary tract infection & $8(8.9)$ & $6(4.9)$ & 0.250 \\
\hline Live abscess & $5(5.6)$ & $7(5.7)$ & 0.995 \\
\hline Urinary tract infection & $7(7.8)$ & $4(3.3)$ & 0.144 \\
\hline Intestinal infection & $4(4.4)$ & $2(1.6)$ & 0.223 \\
\hline Intra-abdominal infection & $3(3.3)$ & $2(1.6)$ & 0.422 \\
\hline Skin infection & $3(3.3)$ & $1(0.8)$ & 0.184 \\
\hline Cerebrospinal fluid & $2(2.2)$ & $0(3.3)$ & 0.098 \\
\hline \multicolumn{4}{|l|}{ Ward at the onset of BSI- No., \% } \\
\hline Intensive care units & $62(68.9)$ & $58(47.5)$ & 0.002 \\
\hline Medical wards & $18(20.0)$ & $36(29.5)$ & 0.116 \\
\hline
\end{tabular}

a During the 12 months preceding infection onset.

${ }^{b}$ During the 30 days preceding infection onset.

${ }^{c}$ During the 72 hours preceding infection onset. 


\begin{tabular}{|llll|}
\hline Variable & Death $(\mathbf{N}=90)$ & Survivors $(\mathbf{N}=122)$ & $P$ value \\
\hline Surgical wards & $10(11.1)$ & $28(23.0)$ & 0.026 \\
\hline Severity at BSI onset- No., & & & \\
\hline APACHE II score $>15$ & $45(50.0)$ & $41(33.6)$ & 0.016 \\
\hline Severe sepsis / septic shock & $60(66.7)$ & $28(23.0)$ & $<0.001$ \\
\hline Therapeutic management- No., \% & & & 0.063 \\
\hline Monotherapy & $7(7.8)$ & $20(16.4)$ & 0.063 \\
\hline Combination therapy & $83(93.3)$ & $102(83.6)$ & 0.001 \\
\hline Combination with high doses of & $30(33.3)$ & $96(78.7)$ & 0.506 \\
\hline carbapenem & & & 0.094 \\
\hline Tigecycline containing regimen & $38(42.2)$ & $46(37.7)$ & 0.240 \\
\hline Aminoglycoside containing regimen & $30(33.3)$ & $28(23.0)$ & \\
\hline Polymyxin B containing regimen & $12(13.3)$ & $16(13.1)$ & \\
\hline Ceftazidime and avibactam containing & $10(11.1)$ & $8(6.6)$ & \\
\hline regimen & & & \\
\hline a During the 12 months preceding infection onset. & & \\
\hline b During the 30 days preceding infection onset. & & \\
\hline c During the 72 hours preceding infection onset. & & \\
\hline
\end{tabular}


Table 5

Multivariate analysis of factors for 28-day mortality in patients with infections caused by CRKP-BSI

\begin{tabular}{|c|c|c|c|}
\hline Variable & $P$ value & OR & $95 \% \mathrm{Cl}$ \\
\hline Congestive heart failure & 0.151 & 7.576 & $0.479-119.913$ \\
\hline Intensive Care Units acquired infection & 0.083 & 3.774 & $0.840-16.969$ \\
\hline Blood purification ${ }^{b}$ & 0.384 & 2.893 & $0.265-31.623$ \\
\hline Mechanical ventilation ${ }^{c}$ & 0.298 & 2.457 & $0.452-13.344$ \\
\hline Corticosteroids use preceding infection onset ${ }^{b}$ & 0.037 & 6.451 & $1.122-37.081$ \\
\hline Inadequate empirical antibiotic therapy & $<0.001$ & 15.006 & $3.704-60.786$ \\
\hline APACHE II score > 15 & 0.294 & 2.189 & $0.506-9.464$ \\
\hline Severe sepsis / septic shock & 0.006 & 8.435 & $1.854-38.385$ \\
\hline Combination with high doses of carbapenem & 0.004 & 0.114 & $0.026-0.508$ \\
\hline${ }^{b}$ During the 30 days preceding infection onset. & & & \\
\hline
\end{tabular}

\section{Discussion}

CRKP-BSI, one of the global public health concerns, has gained much attention for its considerable mortality. In China, a rapid increase of CRKP isolates cases among all KP isolates has been reported since 2010, while the current national average reaches $15.6 \%$ [21]. It was also reported that the incidence of CRKP has ubiquitously increased all over the world [22, 23]. For high CRKP endemic areas such as United States, Greece, Israel, and Italy, the percentage of CRKP-BSI ranged between 18\% and 68\%. [2427]. In the current study, we had an interesting observation of an increase in CRKP-BSI occurrence since 2012, which was significantly higher when comparing average occurrences in China. The possible reason may be attributed to differences in the investigated populations and the severity of the diseases.

To enhance the empirical therapy for CRKP-BSI and to control the emergence, we investigated the risk factors for the occurrence of CRKP-BSI. Our results indicate that there are several factors involved in CRKP infection including hematologic malignancy, ICU acquired infection, hospitalization during the 12 months preceding infection onset, surgery during the 30 days preceding infection onset, inadequate empirical antibiotic therapy, corticosteroids use preceding infection onset and pulmonary source of BSI in univariate analyses. However, only two factors including hematologic malignancy and ICU acquired infection were demonstrated to be independently related to CRKP-BSI by multivariate analysis, which is partly consistent with other reports. $[4,7,28]$. For example, Tian et al. revealed that CRKP-BSIs 
corresponds to ICU acquired infection [4]. Zhang et al. demonstrated that hematologic malignancies were associated with the development of CRKP BSI [12]. Patients with hematologic malignancies usually have frequent long-term hospitalization, undergo more invasive procedures, are exposed with high-grade antibiotics, and have impaired immunological response, which may lead to the development of CRKP-BSI. Therefore, the reinforcement of hygiene protocols in healthcare facilities and rational use of antibiotics should be specially strengthened to prevent the development of CRKP-BSI for patients with hematologic malignancies.

Increasing antibiotic resistance in ICUs is a significant clinical challenge, and we revealed that $56.6 \%$ of CRKP isolates were collected from ICU patients, which was consistent with other reports [26, 29]. It indicates that prevention and control of the occurrence of CRKP-BSI should be focused on ICU. ICU has already been recognized as a factory of creating, disseminating, and amplifying antimicrobial resistance [30]. Nosocomial infection are easily obtained via the airborne and contact transmission of resistant bacteria in the confined environment of ICU [15]. Noteworthy, medical equipment and devices have been demonstrated to be common vectors of CRKP in hospitals, especially in ICUs [29]. The development and transmission of CRKP is much easier in ICU due to the heavy use of the medical equipment and devices for the invasive procedures. Moreover, most ICU patients have relatively serious complications and may be treated with broad spectrum antibiotics or with longer duration of antibiotics use, which may contribute to the induction of carbapenem resistance for KP. To reduce onset of CRKP infections, appropriate controls should be implemented after the admission in ICU, such as active surveillance culture, precautionary isolations, disinfection, initial fitting antibiotic therapy, and relevant antibiotic deescalation[17, 31].

However, previous studies suggested other variables, including surgery within the preceding 90 days, severe chronic comorbidities, previous hospitalizations, indwelling central venous catheter, mechanical ventilation, a nasogastric tube, prior carbapenem administration, and recent exposure to antimicrobials [17, 32-36]. In our study, no antibiotic or invasive procedures were identified as risk factors for CRKP-BSI. This difference may arise from different definition of the infection of CRKP-BSI, the exposure durations to antibiotics, or different patients selected for this research.

In our report, the total mortality rate at 28th day after infection in patients with KP-BSI was $26.1 \%$. Patients with CRKP-BSI had a significantly elevated mortality rate as compared that of CSKP-BSI (42.5 vs. 26.1, $p<0.001$ ), which was consistent with some previous observation [7]. However, other studies contradicted our findings, which showed that the mortality rate between CRKP-BSI and CSKP-BSI was almost the same $[17,24]$. The possible explanation for these conflicting results is that infection-related mortality was associated with several factors, such as host immunity, bacterial virulence, and the efficacy of antibiotics [17].

To further explore the risk factors involved in 28-day mortality, patient characteristics and the therapeutic interventions on CRKP-BSIs were extensively investigated in our study. After adjusting for numerous confounders, various parameters, such as corticosteroids use preceding infection onset, inadequate 
empirical antibiotic therapy, severe sepsis or septic shock and combination therapy with high doses of carbapenem were related to a higher crude 28-day mortality. In agreement with the result by Papadimitriou-Olivgeris et al., we found that the use of corticosteroids preceding infection onset might contribute to the deleterious outcomes of CRKP-BSI patients [37]. Corticosteroids may inhibit a broad range of immune responses, which may have negative effects on infection control and eventually lead to the acceleration of death. Additionally, the well-known association between septic shock and mortality of CR-KP BSI was also observed in our study [27, 38]. However, it is worth mentioning that corticosteroids have been used as adjuncts in the treatment of septic shock according to the guidelines proposed by the Surviving Sepsis Campaign [39]. Whether corticosteroids in the treatment of septic shock due to CP-KP effective or not still needs to be observed and should be validated through randomized controlled trials.

Due to the limited treatment options, inappropriate empirical antibiotic therapy was also demonstrated to be predictive factor for death in CRKP-BSI patients, which is consistent with other studies [38, 40]. Therefore, more attention should be taken to the initial appropriate antibiotic therapy for CRKP-BSI patients. The implementation of antimicrobial stewardship program and regular surveillance of resistance should be strengthened to avoid unnecessary antibiotic exposure.

Regarding the application of protective factors, we showed that there was a significant difference of the 28-day mortality rate between patients treated or not treated with high doses of carbapenem. Although Giannella et al. reported the lack of association between combination therapy with high doses of meropenem and the 14-day mortality, it remained as a protective factor in the multivariate model when adjusted the propensity score [41]. In other studies, various risk factors associated with mortality of patients with CRKP-BSI were identified, including APACHE II score, liver failure, trachea cannula on the day of bacteremia [17], bedridden status, mechanical ventilation, hemodialysis [33], and Pitt bacteremia score [24]. This may be explained by selection bias of study population.

There are several limitations that should be mentioned in this study. Firstly, it was a retrospective analysis of patients from a single center. Clinical data was collected solely according to medical records instead of interviews and clinical examinations of patients with KP-BSI by equally trained doctors. Secondly, we only included cases of K. pneumoniae infection with positive blood cultures. However, cases that were suspected to have KP-BSI but did not have blood samples collected for culture were not included. Therefore, total number of reported KP-BSI incidences can be slightly lower than the actual one. Finally, the lack of more detailed microbiological data, therefore, data related to strain's genotype was not available.

\section{Conclusions}

In conclusion, our results show that in our study hematologic malignancies and ICU-acquired infection were independent risk factors associated with the occurrence of CRKP-BSI. Septic shock, inadequate empirical antimicrobial therapy and corticosteroids use preceding infection onset caused significant increase of mortality rates in CRKP-BSI patients. Combination therapy with high dose carbapenem is 
associated with better outcome. These findings may serve as recommendations for treatments and prevention of CRKP-BSI patients in Changsha, Hunan Province, China.

\section{Abbreviations}

APACHE II: Acute Physiology and Chronic Health Evaluation II; BSIs: bloodstream infections; CCI:

Charlson comorbidity index; $\mathrm{Cl}$ : Confidence interval; COPD: chronic obstructive pulmonary disease; CRKP: carbapenem resistant K. pneumoniae; CRKP-BSI: carbapenem-resistant Klebsiella pneumonia bloodstream infection; E. coli: Escherichia coli; ESBL: extended spectrum beta-lactamase; ICU: intensive care unit; IQRs: Interquartile ranges; KP: Klebsiella pneumoniae; KP-BSI: Klebsiella pneumonia bloodstream infection; OR: Odds ratio

\section{Declarations}

\section{Acknowledgments}

We thank all the colleagues in the Infection Management and Disease Control Department for making this study possible.

\section{Funding}

This work was supported by National Natural Science Foundation of China (81600063 and 81470266) and Natural Science Foundation of Hunan Province, China (2017JJ3484). The grant agency didn't involve in the design of the study, collection, analysis and interpretation of data, and writing or revising the manuscript.

\section{Availability of data and materials}

The datasets generated during the current study are not publicly available due to avoid disclosure the individual privacy of the patients but are available from the corresponding author on reasonable request.

\section{Authors' contributions}

JC, PPH and NRC conceived this study, collected clinical data, interpreted the results, wrote, and revised the manuscript. HM, YL, TS, XX and XH participated in collecting data and data statistics. MM and VS participated in the study design and revised the manuscript. All authors read and approved the final manuscript.

\section{Ethics approval and consent to participate}

This study was approved by the local institutional review board. For this type of study formal consent is not required.

\section{Consent for publication}


Not applicable.

\section{Competing interests}

None of authors declare conflicts of interest relevant to this article.

\section{References}

1. Jiang ZQ, Wang SD, Feng DD, Zhang BX, Mao SH, Wu JN. Epidemiological risk factors for nosocomial bloodstream infections: A four-year retrospective study in China. J Crit Care. 2019;52:92-6.

2. Ocampo AM, Chen L, Cienfuegos AV, Roncancio G, Chavda KD, Kreiswirth BN, Jimenez JN. A TwoYear Surveillance in Five Colombian Tertiary Care Hospitals Reveals High Frequency of Non-CG258 Clones of Carbapenem-Resistant Klebsiella pneumoniae with Distinct Clinical Characteristics. Antimicrob Agents Chemother. 2016;60(1):332-42.

3. Pau CK, Ma FF, Ip M, You JH. Characteristics and outcomes of Klebsiella pneumoniae bacteraemia in Hong Kong. Infectious diseases (London England). 2015;47(5):283-8.

4. Tian L, Tan R, Chen Y, Sun J, Liu J, Qu H, Wang X. Epidemiology of Klebsiella pneumoniae bloodstream infections in a teaching hospital: factors related to the carbapenem resistance and patient mortality. Antimicrobial resistance infection control. 2016;5:48.

5. Nielsen SL, Pedersen C, Jensen TG, Gradel KO, Kolmos HJ, Lassen AT. Decreasing incidence rates of bacteremia: a 9-year population-based study. J Infect. 2014;69(1):51-9.

6. Delle Rose D, Sordillo P, Gini S, Cerva C, Boros S, Rezza G, Meledandri M, Gallo MT, Prignano G, Caccese $\mathrm{R}$, et al. Microbiologic characteristics and predictors of mortality in bloodstream infections in intensive care unit patients: A 1-year, large, prospective surveillance study in 5 Italian hospitals. Am J Infect Control. 2015;43(11):1178-83.

7. Xu L, Sun X, Ma X. Systematic review and meta-analysis of mortality of patients infected with carbapenem-resistant Klebsiella pneumoniae. Ann Clin Microbiol Antimicrob. 2017;16(1):18.

8. Li J, Ren J, Wang W, Wang G, Gu G, Wu X, Wang Y, Huang M, Li J. Risk factors and clinical outcomes of hypervirulent Klebsiella pneumoniae induced bloodstream infections. European journal of clinical microbiology infectious diseases: official publication of the European Society of Clinical Microbiology. 2018;37(4):679-89.

9. Gomez-Simmonds A, Nelson B, Eiras DP, Loo A, Jenkins SG, Whittier S, Calfee DP, Satlin MJ, Kubin CJ, Furuya EY. Combination Regimens for Treatment of Carbapenem-Resistant Klebsiella pneumoniae Bloodstream Infections. Antimicrob Agents Chemother. 2016;60(6):3601-7.

10. Cristina ML, Alicino C, Sartini M, Faccio V, Spagnolo AM, Bono VD, Cassola G, De Mite AM, Crisalli MP, Ottria G, et al. Epidemiology, management, and outcome of carbapenem-resistant Klebsiella pneumoniae bloodstream infections in hospitals within the same endemic metropolitan area. $J$ Infect Public Health. 2018;11(2):171-7. 
11. Hu F, Guo Y, Yang Y, Zheng Y, Wu S, Jiang X, Zhu D, Wang F. Resistance reported from China antimicrobial surveillance network (CHINET) in 2018. European journal of clinical microbiology infectious diseases: official publication of the European Society of Clinical Microbiology. 2019;38(12):2275-81.

12. Zhang Y, Guo LY, Song WQ, Wang Y, Dong F, Liu G. Risk factors for carbapenem-resistant K. pneumoniae bloodstream infection and predictors of mortality in Chinese paediatric patients. BMC Infect Dis. 2018;18(1):248.

13. Schwaber MJ, Klarfeld-Lidji S, Navon-Venezia S, Schwartz D, Leavitt A, Carmeli Y. Predictors of carbapenem-resistant Klebsiella pneumoniae acquisition among hospitalized adults and effect of acquisition on mortality. Antimicrob Agents Chemother. 2008;52(3):1028-33.

14. Chang H, Wei J, Zhou W, Yan X, Cao X, Zuo L, Chen S, Yao K, Huang R, Chen Y, et al: Risk factors and mortality for patients with Bloodstream infections of Klebsiella pneumoniae during 2014-2018: Clinical impact of carbapenem resistance in a large tertiary hospital of China. Journal of infection and public health 2019.

15. Jiao Y, Qin Y, Liu J, Li Q, Dong Y, Shang Y, Huang Y, Liu R. Risk factors for carbapenem-resistant Klebsiella pneumoniae infection/colonization and predictors of mortality: a retrospective study. Pathogens global health. 2015;109(2):68-74.

16. Amit S, Mishali H, Kotlovsky T, Schwaber MJ, Carmeli Y. Bloodstream infections among carriers of carbapenem-resistant Klebsiella pneumoniae: etiology, incidence and predictors. Clinical microbiology infection: the official publication of the European Society of Clinical Microbiology Infectious Diseases. 2015;21(1):30-4.

17. Zheng X, Wang JF, Xu WL, Xu J, Hu J. Clinical and molecular characteristics, risk factors and outcomes of Carbapenem-resistant Klebsiella pneumoniae bloodstream infections in the intensive care unit. Antimicrobial resistance infection control. 2017;6:102.

18. Du M, Xing Y, Suo J, Liu B, Jia N, Huo R, Chen C, Liu Y. Real-time automatic hospital-wide surveillance of nosocomial infections and outbreaks in a large Chinese tertiary hospital. BMC Med Inf Decis Mak. 2014;14:9.

19. Zarkotou O, Pournaras S, Tselioti P, Dragoumanos V, Pitiriga V, Ranellou K, Prekates A, ThemeliDigalaki K, Tsakris A. Predictors of mortality in patients with bloodstream infections caused by KPCproducing Klebsiella pneumoniae and impact of appropriate antimicrobial treatment. Clinical microbiology infection: the official publication of the European Society of Clinical Microbiology Infectious Diseases. 2011;17(12):1798-803.

20. Horan TC, Andrus M, Dudeck MA. CDC/NHSN surveillance definition of health care-associated infection and criteria for specific types of infections in the acute care setting. Am J Infect Control. 2008;36(5):309-32.

21. Hu FP, Guo Y, Zhu DM, Wang F, Jiang XF, Xu YC, Zhang XJ, Zhang CX, Ji P, Xie Y, et al: Resistance trends among clinical isolates in China reported from CHINET surveillance of bacterial resistance, 
2005-2014. Clinical microbiology and infection: the official publication of the European Society of Clinical Microbiology and Infectious Diseases 2016, 22 Suppl 1:S9-14.

22. Moghadampour M, Salari-Jazi A, Faghri J. High rate of carbapenem-resistant Klebsiella pneumoniae detected from hospital equipments in Iran. Acta Microbiol Immunol Hung. 2018;65(4):529-38.

23. Chamieh A, El-Hajj G, Zmerli O, Afif C, Azar E. Carbapenem resistant organisms: A 9-year surveillance and trends at Saint George University Medical Center. Journal of infection and public health 2019.

24. Ben-David D, Kordevani R, Keller N, Tal I, Marzel A, Gal-Mor O, Maor Y, Rahav G. Outcome of carbapenem resistant Klebsiella pneumoniae bloodstream infections. Clinical microbiology infection: the official publication of the European Society of Clinical Microbiology Infectious Diseases. 2012;18(1):54-60.

25. Gomez-Simmonds A, Greenman M, Sullivan SB, Tanner JP, Sowash MG, Whittier S, Uhlemann AC. Population Structure of Klebsiella pneumoniae Causing Bloodstream Infections at a New York City Tertiary Care Hospital: Diversification of Multidrug-Resistant Isolates. J Clin Microbiol. 2015;53(7):2060-7.

26. Alicino C, Giacobbe DR, Orsi A, Tassinari F, Trucchi C, Sarteschi G, Copello F, Del Bono V, Viscoli C, Icardi G. Trends in the annual incidence of carbapenem-resistant Klebsiella pneumoniae bloodstream infections: a 8-year retrospective study in a large teaching hospital in northern Italy. BMC Infect Dis. $2015 ; 15: 415$.

27. Daikos GL, Tsaousi S, Tzouvelekis LS, Anyfantis I, Psichogiou M, Argyropoulou A, Stefanou I, Sypsa V, Miriagou V, Nepka M, et al. Carbapenemase-producing Klebsiella pneumoniae bloodstream infections: lowering mortality by antibiotic combination schemes and the role of carbapenems. Antimicrob Agents Chemother. 2014;58(4):2322-8.

28. Brizendine KD, Richter SS, Cober ED, van Duin D. Carbapenem-resistant Klebsiella pneumoniae urinary tract infection following solid organ transplantation. Antimicrob Agents Chemother. 2015;59(1):553-7.

29. Li Y, Li J, Hu T, Hu J, Song N, Zhang Y, Chen Y. Five-year change of prevalence and risk factors for infection and mortality of carbapenem-resistant Klebsiella pneumoniae bloodstream infection in a tertiary hospital in North China. Antimicrobial resistance infection control. 2020;9(1):79.

30. Hu Y, Ping Y, Li L, Xu H, Yan X, Dai H. A retrospective study of risk factors for carbapenem-resistant Klebsiella pneumoniae acquisition among ICU patients. Journal of infection in developing countries. 2016;10(3):208-13.

31. Daniel Markley J, Bernard S, Bearman G, Stevens MP. De-escalating Antibiotic Use in the Inpatient Setting: Strategies, Controversies, and Challenges. Curr Infect Dis Rep. 2017;19(4):17.

32. Brennan BM, Coyle JR, Marchaim D, Pogue JM, Boehme M, Finks J, Malani AN, VerLee KE, Buckley BO, Mollon N, et al. Statewide surveillance of carbapenem-resistant enterobacteriaceae in Michigan. Infect Control Hosp Epidemiol. 2014;35(4):342-9.

33. Hussein K, Raz-Pasteur A, Finkelstein R, Neuberger A, Shachor-Meyouhas Y, Oren I, Kassis I. Impact of carbapenem resistance on the outcome of patients' hospital-acquired bacteraemia caused by 
Klebsiella pneumoniae. J Hosp Infect. 2013;83(4):307-13.

34. Diaz A, Ortiz DC, Trujillo M, Garces C, Jaimes F, Restrepo AV. Clinical Characteristics of Carbapenemresistant Klebsiella pneumoniae Infections in III and Colonized Children in Colombia. Pediatr Infect Dis J. 2016;35(3):237-41.

35. Akgul F, Bozkurt I, Sunbul M, Esen S, Leblebicioglu H. Risk factors and mortality in the Carbapenemresistant Klebsiella pneumoniae infection: case control study. Pathogens global health. 2016;110(78):321-5.

36. Candevir Ulu A, Kurtaran B, Inal AS, Komur S, Kibar F, Yapici Cicekdemir H, Bozkurt S, Gurel D, Kilic F, Yaman A, et al. Risk factors of carbapenem-resistant Klebsiella pneumoniae infection: a serious threat in ICUs. Medical science monitor: international medical journal of experimental clinical research. 2015;21:219-24.

37. Papadimitriou-Olivgeris M, Fligou F, Bartzavali C, Zotou A, Spyropoulou A, Koutsileou K, Vamvakopoulou S, Sioulas N, Karamouzos V, Anastassiou ED, et al. Carbapenemase-producing Klebsiella pneumoniae bloodstream infection in critically ill patients: risk factors and predictors of mortality. European Journal of Clinical Microbiology Infectious Diseases. 2017;36(7):1125-31.

38. Tumbarello M, Viale P, Bassetti M, De Rosa FG, Spanu T, Viscoli C. Infections caused by KPCproducing Klebsiella pneumoniae: differences in therapy and mortality in a multicentre studyauthors' response. J Antimicrob Chemother. 2015;70(10):2922.

39. Dellinger RP, Levy MM, Rhodes A, Annane D, Gerlach H, Opal SM, Sevransky JE, Sprung CL, Douglas IS, Jaeschke R, et al. Surviving Sepsis Campaign: international guidelines for management of severe sepsis and septic shock, 2012. Intensive care medicine. 2013;39(2):165-228.

40. Fraenkel-Wandel Y, Raveh-Brawer D, Wiener-Well Y, Yinnon AM, Assous MV. Mortality due to blaKPC Klebsiella pneumoniae bacteraemia. J Antimicrob Chemother. 2016;71(4):1083-7.

41. Giannella M, Trecarichi EM, Giacobbe DR, De Rosa FG, Bassetti M, Bartoloni A, Bartoletti M, Losito AR, Del Bono V, Corcione S, et al. Effect of combination therapy containing a high-dose carbapenem on mortality in patients with carbapenem-resistant Klebsiella pneumoniae bloodstream infection. Int J Antimicrob Agents. 2018;51(2):244-8.

\section{Figures}




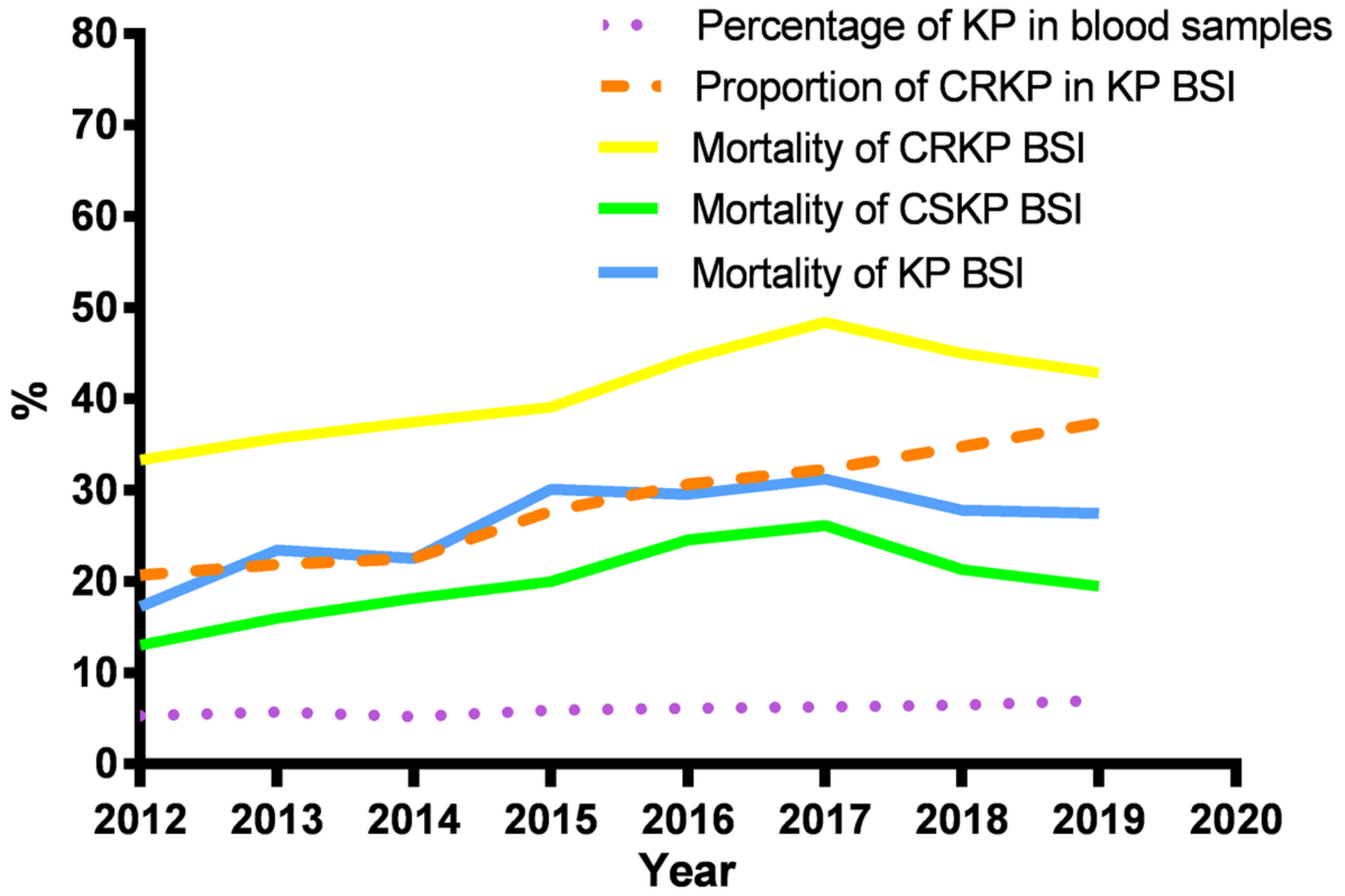

Figure 1

Trends in prevalence and mortality of Klebsiella pneumoniae bloodstream infections in Xiangya Hospital, Hunan Province, China, 2012-2019. KP: Klebsiella pneumoniae; CRKP: Carbapenem-resistant KP; CSKP: Carbapenem-susceptible KP; BSI: bloodstream infection 